\title{
LOCAL ELASTIC CONSTANTS FOR EPOXY-NANOTUBE COMPOSITES FROM MOLECULAR DYNAMICS SIMULATION
}

\author{
S. J. V. Frankland ${ }^{1}$ and T. S. Gates ${ }^{2}$ \\ NASA Langley Research Center \\ Hampton, VA 23681 \\ Materials Research Society Fall Meeting, Boston, MA, November 26-30, 2007
}

\begin{abstract}
A method from molecular dynamics simulation is developed for determining local elastic constants of an epoxy/nanotube composite. The local values of $\mathrm{C}_{11}, \mathrm{C}_{33}, \mathrm{~K}_{12}$, and $\mathrm{K}_{13}$ elastic constants are calculated for an epoxy/nanotube composite as a function of radial distance from the nanotube. While the results possess a significant amount of statistical uncertainty resulting from both the numerical analysis and the molecular fluctuations during the simulation, the following observations can be made. If the size of the region around the nanotube is increased from shells of $1 \AA$ to $6 \AA$ in thickness, then the scatter in the data reduces enough to observe trends. All the elastic constants determined are at a minimum $20 \AA$ from the center of the nanotube. The $\mathrm{C}_{11}, \mathrm{C}_{33}$, and $\mathrm{K}_{12}$ follow similar trends as a function of radial distance from the nanotube. The $\mathrm{K}_{13}$ decreases greater distances from the nanotube and becomes negative which may be a symptom of the statistical averaging.
\end{abstract}

\section{INTRODUCTION}

The inclusion of carbon nanotubes in structural composites for aerospace applications is limited to date, not only by production and processing issues, but also by their lower than expected mechanical performance in polymeric materials. In a composite, the weak interaction between the nanotube and the polymer may be improved by chemical modifications (functionalization) to the carbon nanotube that improve the interface with the polymer. One way to probe the interfacial region is to examine the changes in elastic constants as a function of distance from the carbon nanotube. Elastic constants of polymer nanocomposites can then be determined for representative volume elements (RVE) of the molecular structure by using molecular dynamics simulation to calculate configurational energy changes under mechanical deformation. It is anticipated that the changes observed in the elastic constants are representative of the changes in molecular structure of the polymer in the vicinity of the nanotube, and therefore a measure of the size of the interfacial region. To understand how the nanotube affects the elastic constants of the polymer at the polymer nanotube interface, a broad range of spatial definition is required.

Several theoretical studies have probed the 'interfacial region' of the polymer surrounding the nanotubes to elucidate the effect of the polymer-nanotube interaction on the mechanical behavior. Molecular dynamics (MD) simulations of nanotube pull-out and frictional

\footnotetext{
${ }^{1}$ Senior Research Scientist, National Institute of Aerospace.

${ }^{2}$ Senior Materials Research Engineer, Durability, Damage Tolerance and Reliability Branch.
} 
behavior of nanotubes in polyethylene have demonstrated the slippage of pristine nanotubes in polymer, and the increased resistance to motion when axial load is applied to the nanotube for nanotubes covalently bonded into the polymer [1, 2]. MD simulations have also been used to calculate the stress-strain behavior of nanotubes in polymer matrices [3]. The effect of nanotube functionalization on the elastic constants has been demonstrated by the equivalent continuum method indicating increases in the transverse elastic constants and decreases in the longitudinal elastic constants [4].

The RVEs from MD are on the order of 10,000 atoms or a few nanometers in size. Under deformation, they include energetic changes resulting from the chemical interactions described by the molecular force field. To understand how the nanotube affects the elastic constants of the polymer at the polymer nanotube interface, more spatial definition is required. To describe interfacial mechanical properties, a combined method of micromechanics techniques and molecular dynamics simulation was devised to determine the effects of functionalization on a nanoparticle interface utilizing the radial distribution function of the polymer density away from the nanoparticle [5]. In this study, the interfacial elastic constants were calculated for a functionalized silica particle in polymer for the interface defined by the region between the silica particle and polymer bulk density. The elastic constants were then discerned from the micromechanics method by comparison with elastic constants calculated for the RVE by the equivalent continuum method. An alternative way to calculate the interfacial elastic constants is as a function of radial distance $\mathbf{C}(\mathrm{r})$ from the carbon nanotube directly from the molecular dynamics simulation. The $\mathbf{C}(\mathrm{r})$ has been obtained directly from the atomic structure and the force field of a face centered cubic Lennard-Jones structure [6]. Even for Lennard-Jones interactions, this method is numerically complex. A different method is preferred for polymer-nanotube systems which additionally require consideration of bonding and Coulombic interactions. Some authors have used a method based on the stress fluctuations for polymers $[7,8]$. Both of these works demonstrate that regions of negative moduli are possible within polymer systems.

In the present work, elastic constants are calculated as a function of radial distance $\mathbf{C}(\mathrm{r})$ from the carbon nanotube. These local elastic constants are determined directly from the energies of deformation in the MD simulation for subcomponents of the RVE. The objectives of the present work are first to describe the method, and then to determine the spatial dependence of the elastic constants of the polymer surrounding a carbon nanotube as a function of distance from the nanotube. The spatial dependence is determined for four elastic constants the $\mathrm{C}_{11}, \mathrm{C}_{33}, \mathrm{~K}_{12}$, and $\mathrm{K}_{13}$ where the 3 -direction is the direction of the nanotube. The variation observed in these constants is significant to understanding the extent of the interfacial region in the nanocomposite.

\section{MATERIAL DESCRIPTION}

The representative volume element of the nanocomposite to be simulated contains a $(10,10)$ carbon nanotube in an epoxy matrix (Figure 1). The epoxy matrix is composed of diglycidyl ether of bisphenol A (DGEBA) (Figure 2(a)) cured with diamino-diphenylsulfone (DDS) (Figure 2(b)). The molecular RVE contains about 28000 atoms of cured epoxy and 880 carbon atoms in the nanotube, and is approximately $10 \times 5 \times 5 \mathrm{~nm}$ in size. The epoxy is

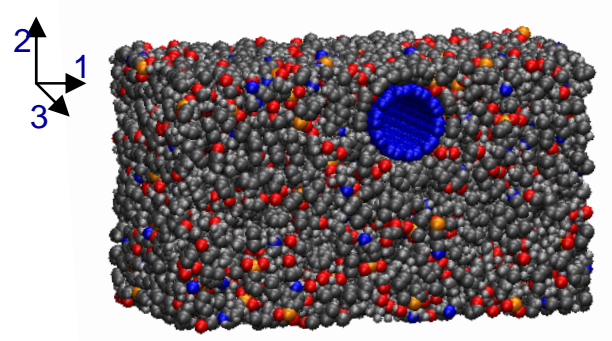

Figure 1. Molecular RVE of epoxy/nanotube composite. 
constructed from one 1:1 network of cured DGEBA/ DDS. It is simulated by using the AMBER force field[9]. Parameters from the literature were added for sulfur [10, 11] and electrostatic charges and molecular geometries were determined from ab initio calculations using the NWCHEM program[12]. The system has periodic boundary conditions in all 3 directions.

The molecular RVE is simulated with

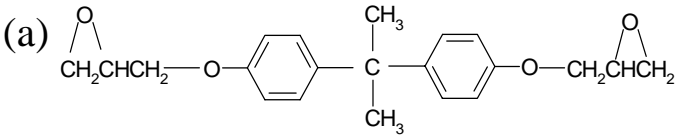

(b)

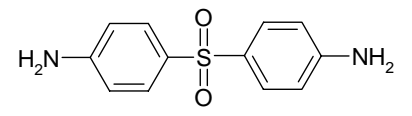

Figure 2. Chemical structures of

(a) DGEBA and (b) DDS. molecular dynamics simulation under NVE (constant number of atoms, volume, and total energy) conditions using velocity rescaling to maintain a constant temperature of $300 \mathrm{~K}$. The system is incrementally deformed every 10000 steps by a positive or negative strain of 0.0025 . Simulations were performed for four different deformations: uniaxial strain in the 1-direction, uniaxial strain in the 3-direction, plane strain in the 1- and 2-directions and plane strain in the 1and 3- directions. Each of these deformations was simulated with positive or negative strain increments for a total of eight simulations. In each case, the strain was referenced to the undeformed system. The four deformations are chosen for determining the following four constants: $\mathrm{C}_{11}, \mathrm{C}_{33}, \mathrm{~K}_{12}$, and $\mathrm{K}_{13}$. The simulations were carried out with DL-POLY[13].

\section{COMPUTATIONAL MODEL}

The local elastic constants of the molecular RVE are obtained as a function of the distance from the nanotube. In each simulation, the configurational energy is calculated per atom. The configurational energy per atom $\Lambda$ includes:

$$
\Lambda=V_{\text {bond }}+V_{\text {angle }}+V_{\text {dihedral }}+V_{L J}+V_{\text {Coulomb }}
$$

The $V_{\text {bond }}$ is one half of the potential energy associated with the chemical bond; the other half belongs to the bonded atom. Similarly $V_{\text {angle }}$ is one half of the valence atom potential energy which is assigned to each of the two atoms connected by the angle, and $V_{\text {dihedral }}$ is one half the dihedral potential energy which is assigned to the first and fourth atoms of the dihedral. Both the Lennard-Jones $\left(V_{L J}\right)$ and the Coulombic potential $\left(V_{\text {Coulomb }}\right)$ energies are included for atom pairs within a cut-off radius of $1.3 \mathrm{~nm}$. The first and fourth atoms of dihedral angles contribute to both $V_{L J}$ and $V_{\text {Coulomb }}$ and are scaled by 0.5 and 0.83 respectively as in the AMBER force field [9]. For the purposes of the analysis, no long range corrections are included for either $V_{L J}$ and $V_{\text {Coulomb }}$, even though these corrections are included in the simulation.

The $\Lambda$ is separated into the configurational energy per ring around the nanotube, and is averaged over the simulation trajectory as a function of strain. Specifically, the $\Lambda$ per ring was calculated from averaging the configurations every 100 time steps from 7000 time steps to 10000 steps after the strain was incremented. The local elastic constants are then determined from the resulting configurational energies per ring from each of the four deformations as a function of both strain and location by the following relations:

$$
\begin{aligned}
& \frac{\Lambda-\Lambda_{0}}{V}=\frac{1}{2} C_{11} \varepsilon_{11}{ }^{2} \\
& \frac{\Lambda-\Lambda_{0}}{V}=\frac{1}{2} C_{33} \varepsilon_{33}{ }^{2}
\end{aligned}
$$




$$
\begin{aligned}
& \frac{\Lambda-\Lambda_{0}}{V}=2 K_{12} \varepsilon_{11} \varepsilon_{22} \\
& \frac{\Lambda-\Lambda_{0}}{V}=2 K_{13} \varepsilon_{11} \varepsilon_{33}
\end{aligned}
$$

All the strain increments are 0.0025. For $\mathrm{K}_{12} \varepsilon_{11}=\varepsilon_{22}$, and for $\mathrm{K}_{13} \varepsilon_{11}=\varepsilon_{33}$. It is assumed that $\Lambda_{0}$ is the configurational energy at zero strain. The local elastic constants for each shell are calculated from numerically fitting $\Lambda$ to a quadratic function of strain. The elastic constants for the molecular RVEs are calculated using the total configurational energies from the same simulations.

\section{RESULTS AND DISCUSSION}

The four elastic constants determined for the molecular RVE are included in Table I. The uncertainty Table I. Elastic Constants for Molecular RVE

reported is the standard error associated with the least squares fit of the configurational energy to the quadratic function of strain. The configurational energy varies during the simulation because the simulation takes place in the NVE ensemble which conserves total energy but allows the configurational energy to

\begin{tabular}{|c|c|}
\hline & $\begin{array}{c}\text { Elastic Constant } \\
(\mathrm{GPa})\end{array}$ \\
\hline $\mathrm{C}_{11}$ & $7.8 \pm 1.1$ \\
\hline $\mathrm{C}_{33}$ & $30 \pm 4$ \\
\hline $\mathrm{K}_{12}$ & $6.0 \pm 0.9$ \\
\hline $\mathrm{K}_{13}$ & $8.5 \pm 1.1$ \\
\hline
\end{tabular}
fluctuate. Therefore at each increment of strain the average configurational energy also varies, and the relationship is not perfectly quadratic. The numerical fit averages the energy fluctuations at different strain levels, and the standard error in the fit reflects the degree of fluctuation. Longer simulations may improve the results by including more configurational averaging.

Figure 3 includes plots of the local elastic constants as a function of radial distance from the center of the nanotube. Results are shown for each $1 \AA$ shell around the nanotube, and the standard error in each fit is reported by the error bars. For the epoxy, the volume used is the volume of the shell, and for the nanotube, the volume includes the thickness of the nanotube and the enclosed volume. The shells are referenced with respect to the nanotube center, and do not change with strain. The influence of the nanotube at about $7 \AA$ is expectedly strong for both the $\mathrm{C}_{33}$ and $\mathrm{K}_{13}$, and it barely contributes at all to $\mathrm{C}_{11}$ and $\mathrm{K}_{12}$. Any trend in epoxy behavior as a function of distance from the nanotube is unclear from these plots. The uncertainties in the elastic constants of the epoxy are much larger than the uncertainty of the elastic constants of the nanotube probably because of the increased mobility of the epoxy atoms relative to the nanotube, and therefore greater ability to sample more local environments. The negative values reflect situations where the energy decreased on average relative to the unstrained configuration. They are more likely the result of scatter in the deviations from positive moduli rather than negative moduli resulting from mechanically unstable parts of the material. Other authors using different methods have observed negative moduli when determining local elastic constants [7, 8].

Figure 4 includes a recalculation of the local elastic constants taking larger intervals away from the nanotube. In Figure 3 the local elastic constants are reported every $1 \AA$, but in Figure 4, they are reported every $6 \AA$. The $6 \AA$ interval was chosen because the first peak in the radial density distribution in the unstrained configuration extends from the edge of the nanotube (about $8 \AA$ ) to $14 \AA$. Therefore, it is reasonable to think of the region of epoxy up to $14 \AA$ as being that of the first 'ring' of atoms surrounding the nanotube. Beyond $14 \AA$, the radial density distribution 

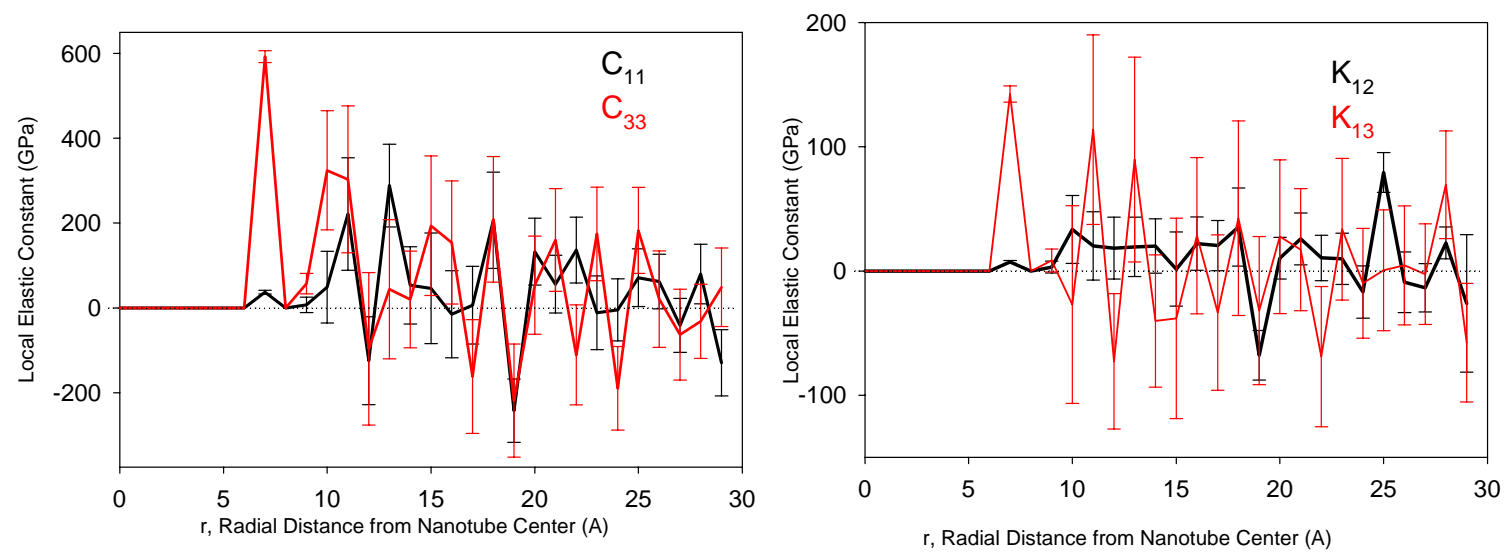

Figure 3. Radial distribution of local elastic constants.

does not indicate clear structure so the averaging was performed for every $6 \AA$ beyond $8 \AA$. This approach avoids some of the negative moduli which are related to the unstable mechanical equilibrium which, in turn, may be related to how much of the group motion of the epoxy atoms around the nanotube is represented. It also substantially reduces the amount of scatter in the data making trends in the elastic constant variation within the RVE visible. All four constants show a minimum at $20 \AA$. Based on the uncertainty in the error bars, this minimum may not be statistically significant. For $\mathrm{C}_{11}$ and $\mathrm{C}_{33}$, the epoxy follows a similar trend with the elastic constant remaining positive. The epoxy $\mathrm{C}_{11}$ and $\mathrm{C}_{33}$ vary by about $70 \mathrm{GPa}$ on average, and the $\mathrm{K}_{12}$ and $\mathrm{K}_{13}$ vary by about 16 and $30 \mathrm{GPa}$ respectively, on average. While the $\mathrm{K}_{12}$ also remains positive out to $26 \AA$, the $K_{13}$ is positive near the nanotube (similar to $K_{12}$ ) and then becomes negative. Ideally, the influence of the nanotube on the surrounding epoxy would be seen as variation in the elastic constant radially from the nanotube. In this unfunctionalized nanotube composite, three of the constants are unaffected by the nanotube's presence in the epoxy to within the statistical uncertainty. If the trend in the $\mathrm{K}_{13}$ behavior is statistically significant, then the nanotube affects the magnitude of $K_{13}$ beyond $26 \AA$. That the $K_{13}$ is negative at 20 and $26 \AA$ may be a symptom of either the statistical averaging or the $6 \AA$ shell thickness. Other authors who have looked at the distribution of elastic moduli around a nanoparticle have found that there are regions within a ring around the nanotube where the modulus is not constant[7, 8]. Further consideration of the size and shape of the region of the epoxy region beyond the nanotube may be needed to obtain a more accurate understanding of the variation of
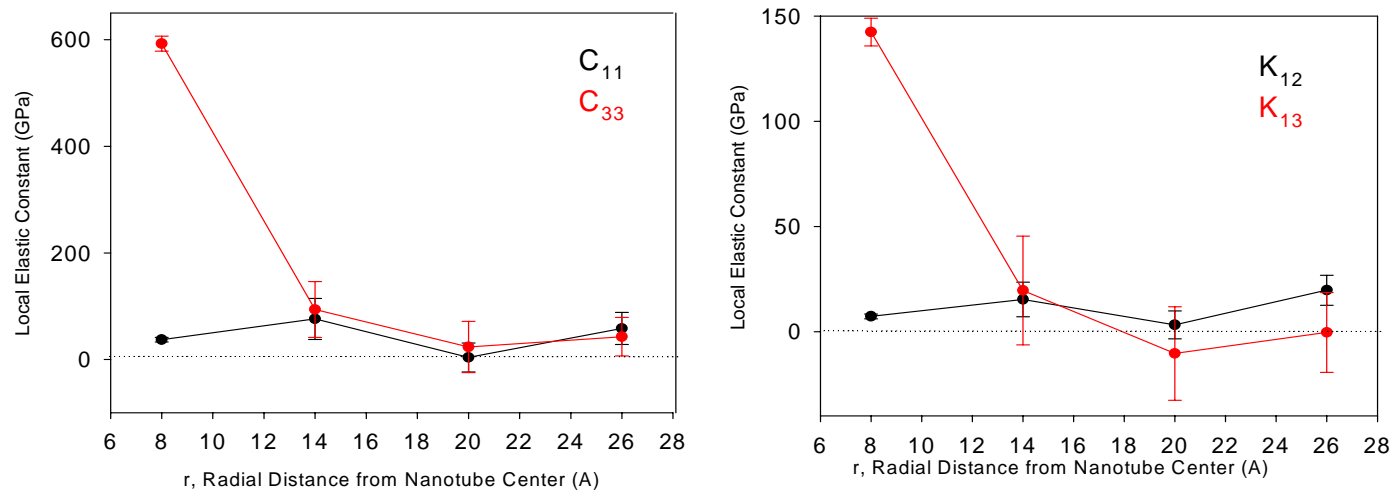

Figure 4. Radial distribution of local elastic constants at every 6A from the nanotube. 
the elastic constant with distance from the nanotube. Also, the small size of the region which can be properly studied with molecular dynamics simulation may be limited given the molecular fluctuations due to mobility of the atoms and thermal fluctuations.

\section{CONCLUSIONS}

A method is developed using the configurational energy within molecular dynamics simulations for determining the local elastic constants of an epoxy/nanotube composite as a function of radial distance from the nanotube. Local $\mathrm{C}_{11}, \mathrm{C}_{33}, \mathrm{~K}_{12}$, and $\mathrm{K}_{13}$ elastic constants are determined. It is found that the results depend in part upon the amount of material included in the analysis and that the results have significant statistical uncertainty of which is partially numerical and partially related to the molecular fluctuations. Increasing the size of the region away from the nanotube from $1 \AA$ shells to $6 \AA$ shells, significantly improves the scatter in the data to the point where trends are visible. All the elastic constants determined are at a minimum $20 \AA$ from the center of the nanotube. The $\mathrm{C}_{11}, \mathrm{C}_{33}$ and $\mathrm{K}_{12}$ follow similar trends as a function of radial distance from the nanotube. The $\mathrm{K}_{13}$ decreases and becomes negative as the distance increases from the nanotube which may be a symptom of the statistical averaging. The potential

of this method to study the elastic behavior of material in the region of the nanotube/epoxy interface rests upon being able to better resolve the degree of fluctuations within the results.

\section{REFERENCES}

1. S.J.V. Frankland, A. Caglar, D.W. Brenner, and M. Griebel, J. Phys. Chem. B 106, 30463048 (2002).

2. $\quad$ S.J.V. Frankland and V.M. Harik, Surf. Sci. 525, L103-L108 (2003).

3. S.J.V. Frankland, V.M. Harik, G.M. Odegard, D.W. Brenner, and T.S. Gates, Comp. Sci. Techn. 63, 1655-1661 (2003).

4. G.M. Odegard, S.J.V. Frankland, and T.S. Gates, AIAA Journal 43, 1828-1835 (2005).

5. $\quad$ G.M. Odegard, T.C. Clancy, and T.S. Gates, Polymer 46, 553-562 (2005).

6. $\quad$ T.J. Delph, Proc. Royal Soc. A 461,1869-1888 (2005).

7. G.J. Papakonstantopoulos, K. Yoshimoto, M. Doxastakis, P.F. Nealey, and J.J. de Pablo, Phys Rev E 72, 031801 (2005).

8. K. Yoshimoto, T.S. Jain, K.V. Workum, P.F. Nealey, and J.J. de Pablo, Phys. Rev. Lett. 93, 175501 (2004).

9. W.D. Cornell, P. Cieplak, C.I. Bayly, I.R. Gould, K.M. Merz, D.M. Ferguson, D.C. Spellmeyer, T. Fox, J.W. Caldwell, and P.A. Kollman, J. Am. Chem. Soc. 117, 51795197 (1995).

10. L.E. Fernandez, A. Ben Altabef, and E.L. Varetti, J. Mol. Struct. 612, 1-11 (2002).

11. N.L. Allinger and Y. Fan, J. Computational Chemistry 14, 655-666 (1993).

12. High Performance Computational Chemistry Group, NWChem, A Computational Chemistry Package for Parallel Computers, Version 4.5 Pacific Northwest National Laboratory, Richland, Washington 99352-0999, USA (2003).

13. DL POLY is a package of molecular simulation routines written by W.Smith and T.R.Forester, copyright The Council for the Central Laboratory of the Research Councils, Daresbury Laboratory at Daresbury, Nr. Warrington (1996). 


\title{
INTERFACIAL ELASTIC CONSTANTS FOR POLYMER-NANOTUBE COMPOSITES FROM MOLECULAR DYNAMICS SIMULATION
}

\author{
S. J. V. Frankland and T. S. Gates \\ NASA Langley Research Center \\ Hampton, VA 23681
}

Materials Research Society Fall Meeting, Boston, MA, November 26-30, 2007

\begin{abstract}
The inclusion of carbon nanotubes in structural composites for aerospace applications is limited to date, not only by production and processing issues, but also by their lower than expected mechanical performance in polymeric materials. In a composite, the weak interaction between the nanotube and the polymer may be improved by chemical modifications (functionalization) to the carbon nanotube that improve the interface with the polymer. One way to probe the interfacial region is to examine the changes in elastic constants as a function of distance from the carbon nanotube. Elastic constants of polymer nanocomposites can then be determined for representative volume elements (RVE) of the molecular structure by using molecular dynamics simulation to calculate configurational energy changes under mechanical deformation. To understand how the nanotube affects the elastic constants of the polymer at the polymer nanotube interface, a broad range of spatial definition is required. In the present work, the elastic constants are calculated as a function of radial distance $\mathbf{C}(\mathrm{r})$ from the carbon nanotube. These interfacial elastic constants are determined directly from the energies of deformation in the MD simulation for subcomponents of the RVE. The objectives of the present work are first to describe the proposed method, and then to determine the spatial dependence of the transversely isotropic elastic constants of the polymer surrounding a carbon nanotube as a function of distance from the nanotube. The method is applied to epoxy/nanotube systems in which the nanotube may be pristine or functionalized.
\end{abstract}

